\title{
Evaluating malingering in cognitive and memory examinations: a guide for clinicians
}

\author{
Derek K. Tracy
}

\section{SUMMARY}

Cognitive and memory testing are a common part of clinical practice, but professional concerns are sometimes raised that the individual being tested might be feigning deficits. Most clinicians have limited experience and training in detecting malingering in such cognitive testing, and the very issue raises considerable ethical dilemmas. Nevertheless, psychiatric work faces ever greater potential for legal scrutiny, and failure to appropriately evaluate potential malingering risks professional embarrassment and distress. There is a need for clinicians to make themselves aware of the ways in which malingered behaviour might be evaluated through the clinical history, the use of routine psychometric testing and, particularly, the use of symptom validity ('malingering') tests. This article describes these factors and gives guidance on the appropriate reporting of findings.

\section{LEARNING OBJECTIVES}

- Better understand the complexities in cognitive assessment where malingering is suspected.

- Understand the types and limitations of the major symptom validity tests.

- Be better prepared to produce documentation and reports stating test findings.

\section{DECLARATION OF INTEREST}

None.

'Anyone who does not feel sufficiently strong in memory should not meddle with lying'

$$
\text { Montaigne, Essays, I, 9, 'Of Liars'. }
$$

Evaluation of memory and cognitive functioning is a common part of practice for many psychiatrists and psychologists. Testing, and clinical work in general, is usually predicated on the assumption that individuals are making honest best efforts in engaging with the task, but clinicians are sometimes faced with the apprehension that an individual is feigning difficulties. This is clearly a concern, particularly if there are current or potential future medico-legal aspects to the work. There are two major domains in which individuals might intentionally feign neurocognitive difficulties: factitious disorders and malingering. Factitious disorders are defined as having (maladaptive) psychological secondary gain and are perhaps best known through Munchausen syndrome. The validity of their diagnostic categorisation and the roles for mental health services are contentious and beyond the remit of this article - the interested reader is referred to the recent review by Bass $\&$ Halligan (2014). Malingering is not a mental illness - therefore it is not 'diagnosed' but, rather, observed or detected - and in DSM-5 (American Psychiatric Association 2013) it has a V-code, being described as:

'The intentional production of false or grossly exaggerated physical or psychological symptoms, motivated by external incentives such as avoiding military duty, avoiding work, obtaining financial compensation, evading criminal prosecution, or obtaining drugs.'

The possible motivations for such behaviours are enormously varied. Identifying potential malingering is clearly important, but it is a difficult area and one in which most clinicians receive little, if any, training. Many clinicians will feel inherently uncomfortable with the prospect, as it runs counter to usual practice; moreover, it can raise troublesome ethical issues and potential damage to the therapeutic relationship, which is normally embedded in principles of trust, respect and confidentiality.

\section{Prevalence}

DSM-5 and ICD-10 (World Health Organization 1992) have reasonably broad definitions of malingering. For clinical work and research the most consistently adhered to criteria are the more specific ones proposed by Slick et al (1999) (Box 1), which have been endorsed by the American Academy of Clinical Neuropsychology in a consensus statement on malingering (Heilbronner 2009). For self-evident reasons it is difficult to confidently determine the rates of malingering, including feigning of cognitive and memory
Derek Tracy is a consultant psychiatrist and the associate clinical director for crisis, in-patient, and rehabilitation services at Oxleas NHS Foundation Trust. He also runs a specialist MSc module in pharmacotherapy at the Institute of Psychiatry, King's College London. Correspondence Dr Derek Tracy, Green Parks House, Princess Royal University Hospital, Orpington, Kent BR6 8NY, UK. Email: derek.tracy@ oxleas.nhs.uk 
BOX 1 Malingering Criteria Checklist

A Presence of a substantial external
incentive
B Evidence from neuropsychological testing
- Definite negative response bias (below
chance on a forced-choice measure of
cognitive functioning)
- Probable response bias on a well-
validated test
- Discrepancies between test data and
known patterns of brain functioning
- Discrepancies between test data and
observed behaviour
- Discrepancies between test data and
reliable collateral reports
- Discrepancies between test data and
documented background history

C Evidence from self-report

- Self-reported history discrepancy with documented history

- Self-reported symptom discrepancy with known patterns of brain functioning

- Self-reported symptom discrepancy with behavioural observations

- Self-reported symptom discrepancy with reports from close informants

- Evidence of exaggerated or fabricated psychological dysfunction

D Behaviours meeting criteria from $B$ or $C$ not fully accounted for by psychiatric, neurological or developmental factors

(Slick 1999)

deficits. If one considers Lipman's (1962) categorisation of the ways individuals can malinger (Box 2), such behaviour is not necessarily a binary characteristic of being 'present' or 'absent': an individual might, for example, be exaggerating genuine difficulties.

An influential and widely cited survey of 33531 clinical reports (Mittenberg 2002) demonstrated probable malingering and symptom exaggeration in: $29 \%$ of personal injury cases (total $n=6371$ ); $30 \%$ of disability cases (total $n=3688$ ); $19 \%$ of criminal cases (total $n=1341$ ); and $8 \%$ of medical cases (total $n=22131$ ). Where mild head injuries were reported, whatever the type of case, probable malingering was detected in $39 \%$. This same work noted some variance depending on the referral source and the nature of the case: in civil cases higher rates of probable malingering were reported in cases referred by defence lawyers and insurers, whereas in criminal cases higher rates were reported in cases referred by prosecutors. Mittenberg et al note that this might be due to a selection bias influencing which cases are referred

B0X 2 Types of malingering

- Invention: creating symptoms where none exist

- Perseveration: describing symptoms that previously occurred

- Exaggeration: magnifying symptom severity

- Transference: attributing real symptoms to a false cause

(Lipman 1962)

for such assessment, whereas Boone et al (2002) estimated a $45 \%$ malingering rate in referrals from legal practices specialising in medical compensation. Exaggeration of symptoms in social security disability examinations in the USA has been estimated to occur in $45.8-59.7 \%$ of cases, and one study (Chafetz 2013) calculated costs due to malingering mental health claimants alone to have been $\$ 20.02$ billion for 2011 .

\section{Assessment of malingering}

\section{The history}

Clinical suspicions of malingering are sometimes aroused in the history taking, particularly if symptoms are diagnostically inconsistent or clinically implausible, or there is clear evidence of secondary gain(s). Symptoms can be disproportionate to expected severity of any objectively documented trauma or postulated illness, or incongruent in pattern with expected physiology. A very early study on the topic by Miller $\&$ Cartlidge (1972) noted that many of those feigning cognitive damage after a mild traumatic brain injury (TBI) performed worse on neuropsychometric testing than those with demonstrable serious TBIs. Further factors that can raise suspicion include:

- temporal anomalies in symptom(s) onset or delayed presentation to appropriate services (including 'referral' by a lawyer)

- discrepancies between various medical records and reports

- unexpected changes reported (or symptoms no longer reported) across sequential clinical interviews

- variance between self-reported symptomatology and objectively observed behaviour.

Of course, none of these factors of itself proves malingering, and it is important to remain objective and non-judgemental; nevertheless, if a clinician has suspicions of malingering then very careful note-keeping is essential to ensure that any such discrepancies are clearly and accurately recorded.

\section{General psychometric testing}

Clinical suspicions might also be aroused during psychometric testing. Most individuals will not have pre-'morbid' baseline testing with which to compare any results, but a careful developmental and educational history - and medical records will provide some implicit assistance. Implausible changes in scores across repeated testing may also raise concerns. It has been argued that feigning 
a consistent pattern of deficits across a range of batteries over time is a considerable challenge (Reitan 1997).

In general, the more detailed the neurocognitive examination, the more confident the clinician can be in detecting patterns of inconsistency or suspicion, although as these are not malingeringspecific tests they can generally only report the deviation from an expected pattern or result. Comprehensive batteries such as the Wechsler Adult Intelligence Scale III (WAIS-III; Wechsler 1997), the Minnesota Multiphasic Personality Inventory-2 (MMPI-2; Butcher 2001) and the Halstead-Reitan Neuropsychological Battery (HRNB; Reitan 1993) have been used in this context, though their complexity means that specialist training is required and the time to administer them is considerable.

The 'simpler' the test, the more difficult it is to detect more subtle patterns of change. Although malingered behaviour might result in grossly abnormal results on tests even as simple as the Mini Mental State Examination (MMSE), it becomes increasingly difficult to reliably interpret such findings, and opinions on them readily lend themselves to challenge or dispute.

A further problem is that research on malingering on general psychometric tests is inevitably hindered, as those who do malinger are unlikely to volunteer to be research participants and the validity of using healthy controls asked to simulate malingering can be questioned.

\section{Specific malingering testing}

The ability to confidently determine malingering through the evaluation of inconsistencies in the history and routine neuropsychometric testing has been fundamentally challenged (Morgan 2009), and it is considered best practice to utilise a purposely designed measurement of malingering if such suspicions or potentials arise (Bush 2005). This is particularly true where there are legal aspects to a case, where a clinician might be called to court to defend any allegation of malingering. In such circumstances, it might prove difficult to justify why specific malingering testing was not undertaken; or indeed to state why no such concerns were raised where the court finds such possibilities exist (Iverson 2003). It is a potentially very difficult environment in which to be: the clinician's clinical rapport or relationship with the tested individual and the room therein for subconscious biases, and their subjective sense of their ability to detect feigning behaviour, can be opened to very detailed forensic legal scrutiny and challenge.

\section{Symptom validity tests - the 'malingering tests'}

Symptom validity tests (SVTs) are specifically designed to evaluate the authenticity of posited symptoms. Most SVTs (but not all) are socalled forced-choice tests that make participants repeatedly choose one of two options - one of which is correct - so that even not understanding the task and random answering should produce a 50\% accuracy rate. Their simplicity means that most individuals should do considerably better than that, even those with neurocognitive deficits, and the worse an individual performs, the greater the likelihood of malingering. A result 'worse than chance' (i.e. a score of $<50 \%$ ) is effectively demonstrating an ability to identify the correct answer by purposely not picking it.

The use of more than one test is recommended because the likelihood that a result is 'true' is greatly enhanced by concordant findings across two different tests, especially if performance is just at a cut-off measure on one test (Larrabee 2003; Nelson 2003). Some guidelines (Bush 2005) encourage informing the testee at the beginning that good effort and honesty are required, and that such factors may be assessed; however, in clinical practice most assessors do not do so (Sharland 2007). There are data to show that, when given last in a battery of tests, participants can find SVTs noticeably easier than other tests, which can raise their suspicions. Whether demographic factors, including cultural or ethnic variations, have an effect on SVT performance remains a generally unanswered question (Bush 2005).

Numerous tests are available, with the major ones described below, and the obvious question arises as to which one(s) to use, particularly as most cost reasonable sums of money to purchase. Hartman (2002) proposed a set of criteria by which to evaluate the quality of malingering tests (Box 3). Interestingly, most tests fail at least one criterion, and many common ones, including the Rey 15-Item Test, fail most criteria.

\section{Test of Memory Malingering (TOMM)}

Dr Tom Tombaugh's TOMM (a name itself surely designed to remain easily in the memory) (Tombaugh 1996) has the most empirical data supporting its validity and is the most widely used SVT (Sharland 2007; Schroeder 2013), particularly among neuropsychologists specialising in personal injury claims (Slick 2004).

Two learning trials are applied to the participant. Each trial consists of 50 sequential line drawings of common objects (the order of presentation 
BOX 3 Criteria for evaluating a malingering test

- Measures willingness to exert basic effort and is insensitive to cognitive dysfunction (specificity and sensitivityl

- Appears to the patient to be a realistic measure of the cognitive modality under study (face validity)

- Measures abilities that are likely to be exaggerated by patients claiming brain damage

- Has a strong normative basis underlying test results to satisfy scientific and legal concerns

- Based on validation studies that include 'normal' participants, patient populations and suspected/verified malingerers

- Difficult to fake or coach

- Easy to administer

- Supported by continuing research

(Hartman 2002)

varies between the trials, but the objects are the same), with each drawing shown for $3 \mathrm{~s}$. In the test period that follows, the participant is shown a further 50 drawings that contain two objects, one of which was on the learning trial and one of which was not. The participant must identify which one they previously saw. An optional memory retention trial (which is identical in structure) can be presented $15 \mathrm{~min}$ later. With each answer, the participant is told whether they are correct or not. Most people score highly on this trial, but a high accuracy rate might cause a malingerer to feel that they are doing unduly 'well' and precipitate a more egregious malingered performance. Scores of less than $45 / 50$ on the second test are considered consistent with malingering, and the worse the performance, the greater the chance that the participant is indeed malingering.

Despite its high performance scores, the TOMM appears to participants to be more difficult than some of the 'simpler' tests such as Rey's, giving it greater face validity as a genuine investigation (Rees 1998). The TOMM is considered to meet Hartman's criteria for validity of a malingering test and its results to be of a calibre admissible as evidence in court. It has been successfully used in a range of neuropsychological conditions and has been shown to be insensitive to the presence of genuine psychological, psychiatric and neurological disability - with the notable exception of severe dementia (Teichner 2004) and to be relatively resistant to different kinds of advance coaching (Jelicic 2011).

\section{Word Memory Test (WMT)}

Like the TOMM, the WMT (Green 1996) is considered to have a high validity, sensitivity and specificity, and to meet Hartman's criteria for a malingering test. Evaluation of these two tests has shown them to be roughly comparable (Greve 2009), although the WMT is more complex in design and delivery.

There are six stages to the test after an initial learning phase: an immediate recognition test; a delayed recognition test, with a 'consistency' score for uniformity of test performance between the immediate recognition and delayed recognition; a multiple choice test; a free recall test; a longdelayed free recall test; and a paired associates test. In the learning phase, 20 strongly associated word pairs (e.g. pig-bacon) are presented twice. This is immediately followed by the immediate recognition test: each of the 20 words is presented with a word with a lower association and the participant is forced to choose which one has been previously seen. Half an hour later, without prior warning, the process of presenting the 20 learned words with (new) lower association pairs is repeated in the delayed recognition stage, again forcing a choice of which one was originally seen. The immediate recognition, delayed recognition and 'consistency' scores account for the main outcome measure of participant test effort. The multiple choice test involves presentation of the initial 40 words with a forced choice of one of eight possible paired words; during the free recall the participant states as many of the words as they can, repeated $20 \mathrm{~min}$ later in the long-delayed free recall; and finally in the paired associates the assessor reads the first word of the initial pairing, which the participant tries to complete.

\section{Forced-Choice Test (FCT)/Digit Memory Test}

Hiscock \& Hiscock's (1989) FCT (also known as the Digit Memory Test) was the first widely used test of memory malingering, and it can be readily and freely constructed. Participants are shown a five-digit number and after a short delay they are asked to identify the number they have just seen from a choice of two, the incorrect answer differing by at least two digits (including either the first or last digit). Three sets of 24 numbers are so delivered, with the delay between presentation of the stimulus number and the forced choice of picking the one of two that matched it increasing from 5 to 10 to $15 \mathrm{~s}$ between the three sets. An early review (Prigatano 1993) demonstrated that those with cerebral dysfunction, even severe, scored 95-100\% accuracy, with those suspected 
of malingering averaging 74\% accuracy. Scores of less than $95 \%$ raise suspicion of malingering. This test is less commonly used these days.

\section{Portland Digit Recognition Test (PDRT)}

The PDRT (Binder 1993) is a variation of the FCT, although it takes considerably longer to deliver. It also has 72 trials, but they are broken into four sets of 18 . Unlike the FCT, the five-digit number is read to the participant (a digit per second) and after a variable gap, a card with two numbers (one above the other) is presented and the participant has to identify the one that represents the number previously spoken. The gaps between stimulus presentation and forced choice are 5 and 15 s for the first two ('easy') sets, and 30 s for the last two ('hard') sets. Furthermore, a distraction task is interleaved within the PDRT in which the participant is told to count backwards between hearing the stimulus and seeing the forced-choice cue: from 20 during the first set, from 50 during the second and from 100 for the last two sets. Cutoff scores for malingering are 19 correct for the easy set, 18 for the hard set and 39 overall.

\section{1-Item Test}

The 21-Item Test (Iverson 1991) is quick to use and relatively cheap to purchase. The assessor reads out 21 words that the participant is asked to free recall. The participant is then presented with word pairs and is forced to choose which one they think they have previously heard. A cut-off score of 9 correct in the force-choiced response is generally used.

\section{Rey 15-Item Test (FIT)}

The FIT (Rey 1964) is one of the oldest, most famous and commonly used (Slick 2004) SVTs, although its validity has been increasingly called into doubt as it does not meet many of Hartman's criteria and has an unacceptably high falsepositive rate (Love 2014). However, its ease of use and scoring, as well as freedom to disseminate without cost, make it popular. In medico-legal situations it should really only be used in addition to other SVTs. Participants are told that they will need to remember 15 different items. They are shown these on a single card (Box 4) for $10 \mathrm{~s}$ before being asked to reproduce them as laid out on the card. Scores below 9 correct are considered indicative of likely poor test engagement.

\section{Coin-in-the-Hand Test}

This is a delightfully simple bedside test, although these attributes potentially open it to significant challenge. The examiner places two outstretched hands, palms up, in front of the participant, with a coin in one. The participant is told to close their eyes and count backwards from 10, opening their eyes when they get to 1 , at which point they have to state which of the (now closed) hands the coin is in. In Kapur's original report (Kapur 1994) two purported malingerers scored equivalent to chance, but five patients with severe amnesia scored 10/10.

\section{Access to, and awareness of, SVTs}

A concern arises as to whether an individual will know that they are receiving a specific test of malingering - and therein, should they be feigning injury, they might produce a 'good' performance to avoid detection. Even more invidious is the concern that individuals might be coached by their legal representative, who may be quite knowledgeable on the principles of neuropsychological assessments (Lees-Haley 1997; Victor 2004). A survey by Essig et al (2001) noted that 21\% of US attorneys prepared their clients on neuropsychometric test content: $19 \%$ on the detection of malingering (with $2 \%$ stating that they reviewed the Rey FIT with their client) and $12 \%$ on what not to divulge in a clinical interview. Although most legal representatives will, reasonably, prepare their client for clinical assessments (as they will for court attendance), clearly it would be an ethical breach of their duty to advise on manipulating clinical tests. Guidelines (Morel 2009) have been prepared for lawyers on this topic and, as in medical practice, the vast majority adhere to their professional ethical standards.

There is some control over inappropriate exposure to SVTs, as most companies that distribute them will not sell them without proof of an appropriate professional registration. Nevertheless, it is not particularly difficult - albeit ethically dubious - to circumvent this to purchase such material; furthermore, a simple internet search or review of articles - including this one - would describe what such tests look like, which

\section{B0X 4 The Rey 15-Item Test}

\begin{tabular}{llll}
\hline A & B & C \\
1 & 2 & 3 & \\
a & b & $c$ \\
0 & $\triangle$ & $\square$ & \\
I & II & III 1964) &
\end{tabular}


would alert the vigilant testee. The American Academy of Clinical Neuropsychology has stated (Heilbronner 2009) that clinicians, though needing to accurately disclose in medico-legal reports or to the court the testing used and its rationale, have a duty to safeguard specific information on SVTs that might preclude their later use through wider dissemination.

\section{The role of the expert and the role of the court}

It is difficult to 'prove' malingering, and there are many factors that could have an impact on performance. Physical factors such as pain, neurological factors such as frontal lobe damage, and psychological factors, including the stress of undergoing psychometric testing, can adversely affect attention, concentration and, thereby, test performance, resulting in apparently disproportionately poor results. This fact, however, underlines the importance of SVTs, as they are designed to be sufficiently simple - although the testee should be unaware of this - that very high scores should be attained even in the presence of significant psychiatric or neuropsychological disorders. However, individuals can purposely not engage with, oppose or actively sabotage testing for reasons besides direct malingering (Cripe 2002), and the presence of secondary gains, which might be very real and valid, and inconsistencies or even frank lies, do not of themselves prove malingering. It is generally most appropriate to discuss test findings in terms of their consistency and validity.

In general, it is easier to state with confidence when testing indicates that there is no evidence - through testing on an SVT - to support a hypothesis that an individual is malingering, although of course this again cannot be proven: an individual feigning a deficit might do so in a very specific area (e.g. a sensory domain or noncognitive psychopathology such as a psychosis) that is unrelated to domains tested by the SVT. As noted earlier, it is of course conceivable that a very well-trained testee would know on which tests to perform well and on which to feign disability, but there are few data to support this in general practice. Whether this will change as testing of malingering becomes more common - which it has - has yet to be seen.

Although a large part of the basis of a clinical judgement is likely to be predicated on performance on SVTs, it is vital to present any evidence in a comprehensive and logical manner:

- background developmental and educational factors
- the history of any alleged injury or illness

- the assessment in hand, which will include the mental state and objective observations on putative cognitive deficits and psychopathology

- any 'non-malingering' neuropsychometry

- clinical and test evidence that would support genuine impairment and an opinion on the strength of such evidence

- inconsistencies and evidence not supporting such impairment, objectively discussed.

This balanced approach is particularly important as, harking back to Lipman's types of malingering, an individual might, for example, have a traumatic brain injury with genuine neurocognitive deficits, with the feigning occurring in their exaggeration rather than their invention. A difficult question is how to feed such information back to the individual tested: a survey by Slick et al (2004) found that most experts tended to discuss test inconsistencies rather than use the term 'malingering'.

\section{Conclusions}

Most SVTs cost money to purchase. However, mindful of Hartman's criteria, failure to use them in appropriate situations could lead to tricky and uncomfortable medico-legal situations, and for clinicians who have reasonable exposure to potential malingering, there is little realistic alternative to their use.

It is important to remember in medico-legal work that it is the job of the court, not the assessing clinician, to determine an individual's credibility as a witness. The clinician must assess, record and report accurately and honestly, but only make statements within the remit of their knowledge and expertise. It is reasonable to note that an SVT was undertaken; to state how it works and why it was done; and to report the individual's scoring on it and how this would ordinarily be interpreted. It is sensible to reiterate that it is for the court to determine the individual's credibility as a witness. The reporting clinician should not attempt to prove that the individual is lying or feigning, or to go beyond their role, for example by trying to link such behaviour with possible secondary gains.

The key points of this article are summarised in Box 5.

\section{References}

American Psychiatric Association (2013) Diagnostic and Statistical Manual of Mental Disorders (5th edn) (DSM-5). APA.

Bass C, Halligan P (2014) Factitious disorders and malingering: challenges for clinical assessment and management. Lancet, 383: 1422-32.

Binder LM (1993) Assessment of malingering after mild head trauma with the Portland Digit Recognition Test. Journal of Clinical and Experimental Neuropsychology, 15: 170-82. 


\section{B0X 5 Key points}

- There is an increasing use of memory tests in medicolegal situations

- There are many reasons for suboptimal test performance

- Clinical suspicions and general psychometric tests are often not sufficient in evaluating possible malingering: (at least one) symptom validity test should be used

- It is easier to state with confidence when an individual's results are not consistent with malingering

- Other confounders can negatively alter results and findings should be reported with caution

- Ultimately the credibility of a witness, and the charge of malingering, is for the court to decide

Boone KB, Lu P, Back C, et al (2002) Sensitivity and specificity of the Rey Dot Counting Test in patients with suspect effort and various clinical samples. Archives of Clinical Neuropsychology, 17: 625-42.

Bush SS, Ruff RM, Troster Al, et al (2005) Symptom validity assessment: practice issues and medical necessity NAN Policy \& Planning Committee. Archives of Clinical Neuropsychology, 20: 419-26.

Butcher JN, Graham JR, Ben-Porath YS, et al (2001) Minnesota Multiphasic Inventory-2: Manual for Administration, Scoring and Interpretation (Revised Edition). University of Minnesota Press.

Chafetz M, Underhill J (2013) Estimated costs of malingered disability. Archives of Clinical Neuropsychology, 28: 633-9.

Cripe LI (2002) Malady versus malingering: a tricky endeavor. In Functional Disorders (eds ND Zasler, MF Martelli): 95-112. Hanley and Belfus.

Essig SM, Mittenberg W, Petersen RS, et al (2001) Practices in forensic neuropsychology: perspectives of neuropsychologists and trial attorneys. Archives of Clinical Neuropsychology, 16: 271-91.

Green P, Allen LM, Astner K (1996) The Word Memory Test: A User's Guide to the Oral and Computer Administered Forms. Cognisyst Inc.

Greve KW, Binder LM, Bianchini KJ (2009) Rates of below-chance performance in forced-choice symptom validity tests. Clinical Neuropsychologist, 23: 534-44.

Hartman DE (2002) The unexamined lie is a lie worth fibbing: neuropsychological malingering and the Word Memory Test. Archives of Clinical Neuropsychology, 17: 709-14.

Heilbronner RL, Sweet JJ, Morgan JE, et al (2009) American Academy of Clinical Neuropsychology Consensus Conference Statement on the neuropsychological assessment of effort, response bias, and malingering. Clinical Neuropsychologist, 23: 1093-129.

Hiscock M, Hiscock CK (1989) Refining the forced-choice method for the detection of malingering. Journal of Clinical and Experimental Neuropsychology, 11: 967-74.

Iverson GL (2003) Detecting malingering in civil forensic evaluations. In Handbook of Forensic Neuropsychology (eds AM Horton, LC Hartlage): 137-79. Springer Publishing Company.

Iverson GL, Franzen MD, McCracken LM (1991) Evaluation of an objective assessment technique for the detection of malingered memory deficits. Law and Human Behavior, 15: 667-76.

Jelicic M, Ceunen E, Peters MJ, et al (2011) Detecting coached feigning using the Test of Memory Malingering (TOMM) and the Structured Inventory of Malingered Symptomatology (SIMS). Journal of Clinical Psychology, 67: 850-5.

Kapur N (1994) The coin-in-the-hand test: a new 'bed-side' test for the detection of malingering in patients with suspected memory disorder. Journal of Neurology, Neurosurgery and Psychiatry, 57: 385-6.
Larrabee GJ (2003) Detection of malingering using atypical performance patterns on standard neuropsychological tests. Clinical Neuropsychologist, 17: 410-25

Lees-Haley PR (1997) Attorneys influence expert evidence in forensic psychological and neuropsychological cases. Assessment, 4: 321-4.

Lipman FD (1962) Malingering in personal injury cases. Temple Law Quarterly, 35: 141-62.

ove CM, Glassmire DM, Zanolini SJ, et al (2014) Specificity and false positive rates of the Test of Memory Malingering, Rey 15-Item Test, and Rey Word Recognition Test among forensic inpatients with intellectual disabilities. Assessment, 26 Mar, doi: 10.1177/1073191114528028.

Miller H, Cartlidge N (1972) Simulation and malingering after injuries to the brain and spinal cord. Lancet, 1: 580-5.

Mittenberg W, Patton C, Canyock EM, et al (2002) Base rates of malingering and symptom exaggeration. Journal of Clinical and Experimental Neuropsychology, 24: 1094-102.

Morel KR (2009) Test security in medicolegal cases: proposed guidelines for attorneys utilizing neuropsychology practice. Archives of Clinical Neuropsychology, 24: 635-46.

Morgan JE, Sweet JJ (eds) (2009) Neuropsychology of Malingering Casebook. Psychology Press.

Nelson NW, Boone KB, Dueck A, et al (2003) Relationship between eight measures of suspect effort. Clinical Psychologist, 17: 263-72.

Prigatano GP, Amin K (1993) Digit Memory Test: unequivocal cerebral dysfunction and suspected malingering. Journal of Clinical and Experimental Neuropsychology, 15: 537-46.

Rees LM, Tombaugh TN, Gansler DA, et al (1998) Five validation experiments of the Test of Memory Malingering (TOMM). Psychological Assessment, 10: 10-20.

Reitan RM, Wolfson D (1993) The Halstead-Reitan Neuropsychological Test Battery: Theory and Clinical Interpretation (2nd edn). Neuropsychology Press.

Reitan RM, Wolfson D (1997) Consistency of neuropsychological test scores of head-injured subjects involved in litigation compared with head-injured subjects not involved in litigation: development of the Retest Consistency Index. Clinical Psychologist, 17: 69-76.

Rey A (1964) L'Examen Clinique en Psychologie [Clinical Tests in Psychology]. Presses Universitaires de France.

Schroeder RW, Buddin WH Jr, Hargrave DD, et al (2013) Efficacy of Test of Memory Malingering Trial 1, Trial 2, the Retention Trial, and the Albany Consistency Index in a criterion group forensic neuropsychological sample. Archives of Clinical Neuropsychology, 28: 21-9.

Sharland MJ, Gfeller JD (2007) A survey of neuropsychologists' beliefs and practices with respect to the assessment of effort. Archives of Clinical Neuropsychology, 22: 213-23.

Slick DJ, Sherman EM, Iverson GL (1999) Diagnostic criteria for malingered neurocognitive dysfunction: proposed standards for clinical practice and research. Clinical Neuropsychologist, 13: 545-61.

Slick DJ, Tan JE, Strauss EH, et al (2004) Detecting malingering: a survey of experts' practices. Archives of Clinical Neuropsychology, 19: 465-73.

Teichner G, Wagner MT (2004) The Test of Memory Malingering (TOMM): normative data from cognitively intact, cognitively impaired, and elderly patients with dementia. Archives of Clinical Neuropsychology, 19: 455-64.

Tombaugh TN (1996) The Test of Memory Malingering. Multi-Health Systems.

Victor TL, Abeles N (2004) Coaching clients to take psychological and neuropsychological tests: a clash of ethical obligations. Professional Psychology: Research and Practice, 35: 373-9.

Wechsler D (1997) Wechsler Adult Intelligence Scale III. Psychological Corporation.

World Health Organization (1992) The ICD-10 Classification of Mental and Behavioural Disorders: Clinical Description and Diagnostic Guidelines. WHO

\section{MCO answers}

1 e $\quad 2$ c $\quad 3$ b $\quad 4$ b

$5 a$ (i) b(ii) $c$ (ii) $d$ (i) e (i) 


\section{MCOs}

3 Types of malingering include:

Select the single best option for each question stem

1 Malingering:

a is a medical diagnosis

b is proven by an expert report

c is reliably detected through a careful history

$\mathrm{d}$ is reliably picked up through an expert clinical opinion

e is ultimately a decision for the court(s).

2 All symptom validity tests (SVTs):

a produce a $50 \%$ accuracy score if answered randomly

b should meet Lipman's criteria

c produce probabilistic outcomes

d should be undertaken at the end of a test battery

e should be disclosed to the testee.

b can only be indirectly inferred a projective malingering

b transference malingering

c factitious malingering

d dissociative malingering

e malingering by proxy.

\section{Rates of malingering:}

a are determined by court convictions

c are greater in criminal than in civil cases

d are more than $50 \%$ in medical compensation cases

e are approximately $15 \%$ in civil cases involving traumatic brain injuries.
5 To prove you have read this article, and are not malingering about your CPD, pick the actual SVT out of each of the following pairs (scores $<80 \%$ indicate malingering):

a (i) Rey's 15-Item Test

(ii) Bird-in-the-Hand Trick

b (i) Oregon Digit Test

(ii) 21-Item Test

c (i) Thompson's Malingerers' Memory

(ii) Coin-in-the-Hand Test

d (i) Forced-Choice Test

(ii) World Memory Test

e (i) Test of Malingering Memory

(ii) Tracy's Malingering Test. 